\title{
INTEGRAR SALA DE AULA E REDE SOCIAL: COMPARTILHANDO UMA EXPERIÊNCIA COM PERSPECTIVA GEOMÉTRICA!
}

\section{INTEGRATE THE CLASSROOM AND SOCIAL NETWORK: SHARING AN EXPERIENCE WITH PERSPECTIVE GEOMETRIC!}

\section{Organdi Mongin Rovetta, Sandra Aparecida Fraga da Silva}

Instituto Federal do Espírito Santo

E-mail: organdimongin@hotmail.com, sandrafraga7@gmail.com

\section{Resumo}

Este artigo tem por objetivo identificar habilidades do pensamento geométrico de alunos do ensino médio e suas formas de interlocução explicitadas durante o processo de interação na resolução de atividades sobre perspectiva tanto em sala de aula como no ambiente virtual. Trata-se de um recorte de uma pesquisa de mestrado que propôs a utilização pedagógica das redes sociais como um espaço complementar à sala de aula. Selecionamos atividades realizadas em sala de aula e na rede social, que apresentam habilidades do pensamento geométrico construídas por meio da interação de alunos do 30 ano do ensino médio durante sua resolução. Utilizamos como suporte a teoria de Van Hiele, as concepções de Angel Gutiérrez sobre visualização e aos trabalhos de Marcelo Bairral sobre interações em ambientes virtuais. Percebemos que é possível estabelecer uma conexão entre sala de aula e rede social interferindo positivamente na aprendizagem dos alunos. Além do mais, as interações desenvolvidas no ambiente virtual permitem ao professor analisar o desenvolvimento do raciocínio matemático dos alunos.

Palavras-chave: sólidos geométricos. visualização. redes sociais. interação.

\section{Abstract}

This article aims to identify the geometric thinking of high school students skills and forms of dialogue explained during the process of interaction in solving perspective on activities both in the classroom and the virtual environment. It is a cutout of a Master thesis that proposed the pedagogical use of social networks as a complementary space to the classroom. Selected activities performed in the classroom and on the social network, which feature geometric thinking skills built through the interaction of the 3rd year of middle school students during their resolution. We used as support the theory of Van Hiele, the Angel Gutierrez views about display and Marcelo Bairral work on interactions in virtual environments. We realize that you can establish a connection between the classroom and social network interfering positively on student learning. Moreover, the interactions developed in the virtual environment allows the teacher to analyze the development of mathematical reasoning of the students.

Palavras-chave: geometric solids. preview. social networks. interaction. 


\section{INTRODUÇÃO}

Em tempos de novas mídias, vale refletir sobre como elas podem se tornar aliadas ao processo educativo. Fazer uso adequado dessas tecnologias na educação não é uma tarefa tão simples. As escolas precisam mais do que estar equipadas com laboratórios de informática, necessitam que os professores utilizem metodologias adequadas para fazerem uso desse tipo de tecnologia.

Desde a implantação dos laboratórios de informática nas escolas, algumas fases das tecnologias digitais marcaram a educação matemática. Borba, Silva e Gadanidis (2014) chamam a atenção para a atual fase que estamos vivendo, caracterizada pela internet rápida. A produção de conhecimento a distância ganha cada vez mais espaço e novas formas de interação começam a ser exploradas. Nesse contexto, surgem novos ambientes virtuais, as redes sociais.

O dinamismo que as redes sociais possibilitam pode ser destacado como um fator positivo da utilização desses ambientes na educação. A rede social Facebook, por exemplo, oferece uma plataforma de interação gratuita com seus recursos extremamente funcionais, permitindo a troca de informações e experiências em tempo real. Além do mais, por meio da utilização de redes sociais, o aluno pode multiplicar o conhecimento para sua teia social, e o professor, por sua vez, utilizar a plataforma como um canal para compartilhar informações e aprofundar os temas discutidos em sala de aula.

Foi nessa perspectiva que se desenvolveu a atividade apresentada neste artigo, que trata de um recorte da pesquisa de mestrado intitulada Interações em sala de aula e em redes sociais no estudo de sólidos geométricos no ensino médio (ROVETTA, 2015). O foco da referida pesquisa foi a utilização pedagógica das redes sociais, especificamente o Facebook, como um espaço complementar a sala de aula visando estabelecer uma relação entre o ambiente presencial (sala de aula) e o ambiente virtual (rede social), a fim de propiciar interação entre os envolvidos, além de oportunizar a utilização de outros recursos multimídias. 
A possibilidade de integração de mídias, bem como o fato de mais de $90 \%$ dos alunos envolvidos terem perfil na rede social Facebook, nos levaram a escolhê-la para a realização da pesquisa. Além do mais, ser um ambiente de que os alunos já fazem uso, logo já conhecem os recursos disponíveis na plataforma, facilita a realização das atividades propostas e a interação. Criar outro ambiente utilizando uma plataforma própria possivelmente não seria tão dinâmico quanto utilizar um ambiente já conhecido, porém com uma nova finalidade.

Dessa forma, selecionamos uma das atividades realizadas pelos alunos no ambiente virtual, para discutirmos neste artigo. Assim, nosso objetivo é identificar habilidades do pensamento geométrico de alunos do ensino médio e suas formas de interlocução explicitadas durante o processo de interação na resolução de atividades sobre perspectiva tanto em sala de aula como no ambiente virtual.

\section{COMENTANDO SOBRE INTERAÇÕES EM AMBIENTES VIRTUAIS}

As tecnologias educacionais mais recentes compreendem vários recursos e a utilização da internet, dos softwares, e dos espaços virtuais de comunicação (blogs, chats, redes socais). Esses espaços são propícios para o aprendizado matemático que se desenvolve por meio de uma relação mútua entre discurso e interação (BAIRRAL, 2007). Ainda segundo o autor, uma das características atribuída à informática educativa é a integração de mídias, pois as tecnologias da informação e comunicação podem integrar várias outras compondo assim um novo cenário para o processo de ensino e aprendizagem.

Sobre os ambientes virtuais de aprendizagem, de acordo com Duart e Sangrá (1999 apud BAIRRAL, 2007), eles devem possibilitar flexibilidade, interatividade, inserção e vinculação na comunidade virtual constituída e permitir aos envolvidos o acesso a materiais e demais fontes de recursos disponíveis na rede. Bairral (2007) destaca, ainda, que, em seus projetos, 
a aprendizagem matemática é desenvolvida em ambientes virtuais onde os interlocutores interagem colaborativamente com diferentes artefatos.

A produção escrita que ocorre nos ambientes virtuais é constituída por interlocutores que interagem por meio de práticas discursivas, as interlocuções. Bairral e Powell (2013) destacam a importância das interlocuções como uma estratégia de análise do desenvolvimento das ideias matemáticas dos envolvidos. A análise das interlocuções, por Bairral e Powell (2013), surgiu com base em Powell (2006), que analisa as práticas discursivas dos alunos em suas trocas conversacionais. Para ele, as ideias e modos de raciocínio podem aflorar da interação discursiva de interlocutores. Para analisar as interações discursivas, Davis (1997 apud Powell, 2006) estabelece quatro propriedades das interlocuções, na qual recorremos para analisar as interações online dos alunos no Facebook. São elas:

a) Avaliativa: o interlocutor se mantém avaliador e não participativo. As afirmações são apresentadas de forma crítica, com julgamentos pontuais como certo ou errado.

b) Informativa: o interlocutor menciona alguma informação para satisfazer ou gerar uma pergunta, mas sem manifestar seu julgamento.

c) Interpretativa: o interlocutor se posiciona de maneira a entender e interpretar o que outro interlocutor disse.

d) Negociativa: o interlocutor e seu parceiro interagem mutuamente, fazendo questionamentos e participando coletivamente na busca de uma solução.

Defendemos que analisar interlocuções em discursos online possibilita acompanhar as mudanças que ocorrem durante a construção do conhecimento matemático por parte dos alunos ao participarem de atividades no ambiente virtual. Além disso, permite também analisar como os sujeitos compartilham significados e conceitos e, no nosso caso, como esses alunos evidenciam diálogos sobre sólidos geométricos. Por isso é importante pensarmos neste processo de construção do pensamento geométrico. 


\section{POSTANDO SOBRE O ENSINO DE GEOMETRIA}

Segundo Nacarato e Santos (2014) o ensino da geometria no Brasil passou por várias fases, sendo até 1960 baseada numa proposta tradicional dos estudos de Euclides, e entre 1970 e 1980 influenciada pelo Movimento da Matemática Moderna, que valorizou mais as estruturas algébricas. Essas ações e outros fatores que não abordamos neste trabalho causaram um abandono da geometria na escola básica (MIGUEL, FIORENTINI, MIORIM, 1992). A partir da década de 1990 houve um aumento em pesquisas sobre geometria, os Parâmetros Curriculares Nacionais - PCN - e o Plano Nacional do Livro Didático contribuíram para uma nova fase que possibilitou modificações na abordagem de geometria no Brasil. Mas, apesar das mudanças que já ocorreram, o ensino de geometria no Brasil ainda é deficitário, pois a forma como os conceitos são explorados nem sempre favorece a sua aprendizagem.

O processo de aprendizagem em geometria passou a ser foco de análise e discussão de pesquisas ao longo destes períodos. Nasser e Tinoco (2011) explicam que, na década de 1950, os professores holandeses Pierre Van Hiele e Dina Van Hiele-Geoldof perceberam que seus alunos tinham dificuldades em resolver tarefas de geometria, enquanto apresentavam um bom desempenho em outros tópicos da Matemática. A partir de uma investigação eles desenvolveram uma teoria para explicar o desenvolvimento do raciocínio em geometria, a teoria de Van Hiele. Essa teoria considera que há cinco níveis de desenvolvimento do pensamento geométrico: visualização, análise, dedução informal, dedução formal e rigor.

Chamamos a atenção para o nível inicial dessa teoria, pois ao refletirmos sobre o ensino de geometria, percebemos que ainda ocorre a predominância dos termos algébricos em detrimento de atividades que exploram a visualização.

Neste trabalho a visualização na matemática é um tipo de atividade de raciocínio baseada no 
uso de elementos visuais, seja mental ou físico, realizado para resolver problemas, ou provar propriedades (GUTIÉRREZ, 1996). As imagens visuais dos objetos que são manipulados em atividades de visualização se realizam, segundo Gutierrez (1996), por dois tipos de processo: o processamento visual, que diz respeito à conversão da informação abstrata em imagens visuais e também ao processo de transformação de uma imagem visual já formada em outra e a interpretação de informação figurativa; a interpretação figurativa que seria o inverso do processamento visual e trata-se, portanto, do processo de compreensão e interpretação de representações visuais.

Gutiérrez (1991) chama a atenção para as habilidades utilizadas pelos indivíduos para a criação e o processamento de imagens visuais. Del Grande (1990 apud GUTIÉRREZ, 1991) apresenta uma relação das habilidades que podem integrar a percepção espacial de um indivíduo, a saber:

a) coordenação motriz dos olhos - seguir com os olhos o movimento dos objetos de forma ágil e eficaz;

b) identificação visual - reconhecer uma figura num contexto maior, ou seja, quando ela é formada por várias partes, por exemplo, identificar uma determinada forma num mosaico;

c) conservação da percepção - reconhecer que um objeto mantém sua forma, mesmo quando girado;

d) reconhecimento de posições no espaço - relacionar a posição do objeto com um ponto de referência;

e) reconhecimento das relações espaciais - identificar características de relações entre diversos objetos situados no espaço;

f) discriminação visual - comparar vários objetos identificando semelhanças e diferenças;

g) memória visual - recordar características visuais e posições de um conjunto de objetos que, em um dado momento, estavam à vista.

Dessa forma, compreendemos que dentro de cada nível apresentado na teoria de Van Hiele, 
também há um conjunto de habilidades e que elas são construídas pelos alunos no decorrer do processo de maneira que, conforme indicam Gutiérrez, Jaime e Fortuny (1991), é possível adquirir habilidades de um nível superior e ainda estar em processo de construção de determinadas habilidades do nível anterior. Portanto, compreendemos que os níveis não são excludentes, o importante é compreender a construção do pensamento geométrico e quais habilidades são desenvolvias ao longo deste processo pelo indivíduo.

\section{COMPARTILHANDO A PROPOSTA DE TRABALHO}

A atividade aqui apresentada foi realizada em uma escola estadual localizada em Iconha/ES e envolveu 89 alunos distribuídos em quatro turmas de 3a série do Ensino Médio, cuja professora de matemática é a primeira autora deste artigo.

Para a prática, foi criado um grupo chamado "sólidos geométricos" na rede social Facebook, no qual durante aproximadamente 40 dias foram desenvolvidas as atividades, totalizando 16 aulas presenciais de matemática nos meses de maio, junho e julho de 2014.

A ideia da prática foi integrar dois ambientes de aprendizagem: sala de aula (ambiente presencial) e grupo criado no Facebook (ambiente virtual). Este último, caracterizado como uma extensão da sala de aula com a finalidade de interação e comunicação, tendo como base as discussões realizadas presencialmente em aulas de matemática. As aulas presenciais foram conduzidas para que os alunos visualizassem e analisassem sólidos geométricos por meio de atividades que utilizavam material concreto, softwares de visualização, trabalhos em grupo, construções geométricas, dentre outros.

De forma paralela ao ambiente presencial, no ambiente virtual foram desenvolvidas atividades diferenciadas envolvendo sites que possibilitavam trabalhar com geometria dinâmica, acesso a vídeos, resolução e discussão de problemas envolvendo visualização, postagem de materiais produzidos, etc. É importante destacar que cada uma dessas 
atividades era interligada com as atividades realizadas em sala de aula. Algumas discussões começavam em sala de aula e se estendiam para o ambiente virtual; outras tinham como base uma postagem no ambiente virtual cuja discussão se estendia para sala de aula.

Selecionamos uma atividade desenvolvida em sala de aula (Perspectiva), bem como a sua correspondente desenvolvida no ambiente virtual (discussão de problema de visualização), que apresentamos na sequência.

\section{NAVEGANDO PELAS ATIVIDADES}

As discussões sobre perspectiva começaram com a construção de blocos utilizando o material dourado. Os alunos deveriam fazer as representações ortogonais superior, frontal e lateral do bloco construído. Na aula seguinte, com o auxílio de uma apresentação em PowerPoint, foi possível discutir alguns tipos de perspectivas e explorar a isométrica. $\mathrm{Na}$ ocasião, os alunos aprenderam a utilizar a malha isométrica para representar poliedros no plano.

Dando continuidade ao estudo sobre perspectiva, algumas atividades, retiradas do livro Vendo e entendendo os poliedros, de Kaleff (2003), foram organizadas em uma apostila e trabalhadas durante aproximadamente três aulas de 55 minutos. Os alunos trabalharam em grupo e utilizaram o material dourado como apoio manipulativo e visual. As atividades da apostila consistiam em fazer representações em perspectiva isométrica tendo como base o poliedro construído com material dourado ou mesmo a representação desse sólido feita no plano. Atividades envolvendo projeção ortogonal, ou vistas, também foram abordadas, bem como a representação cotada de um sólido construído com junção de blocos quadrados. Segundo Carvalho (2010), o termo representação cotada é utilizado por Kaleff (2003) para as representações que se recorre a números que indicam quantos cubos existem em cada direção perpendicular ao observador. Já para Gutiérrez (1998), esse tipo de representação é denominado de projeção ortogonal codificada. 
Além de abordar as representações em perspectiva, as atividades também tiveram como objetivo desenvolver habilidades visuais importantes para o desenvolvimento do pensamento geométrico. A figura 1 mostra algumas atividades realizadas pelos alunos.

Figura 1. Realização de tarefas da apostila.

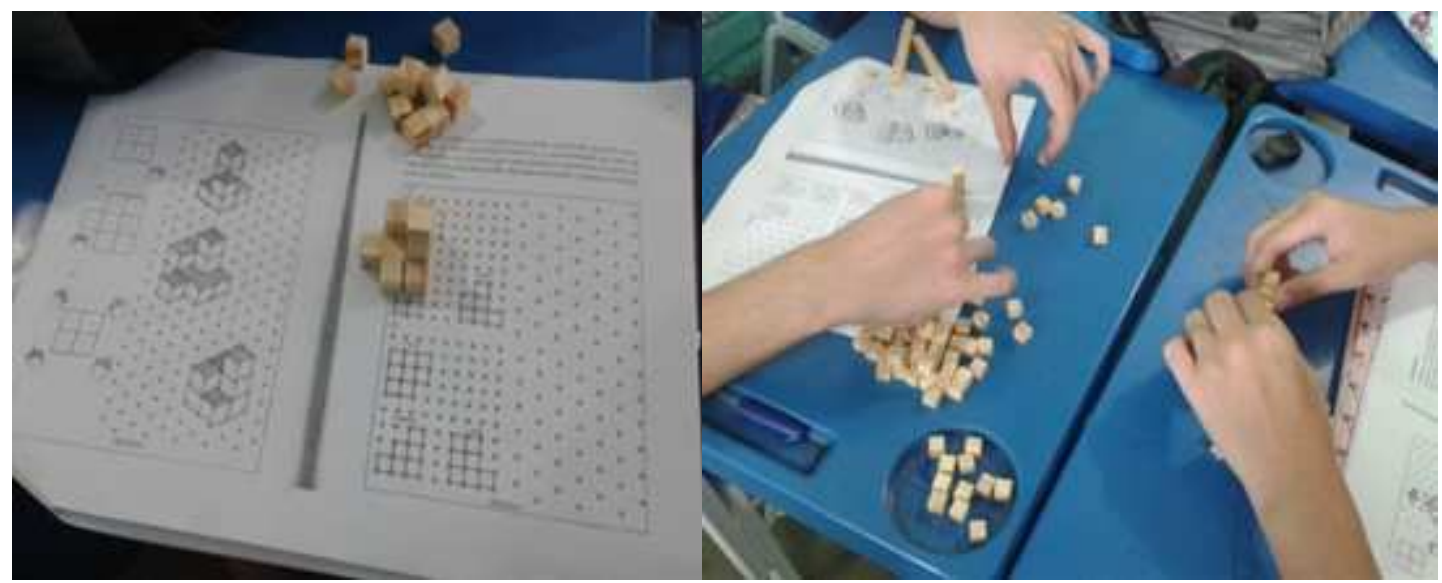

Fonte: dados da pesquisa.

As atividades desenvolvidas se justificam com os estudos de Gutierrez sobre representação de sólidos no plano. Gutiérrez (1998) investiga as formas de orientar os alunos nos diferentes métodos de representação dos objetos tridimensionais, destacando a necessidade do aluno manipular o sólido para vê-lo em uma posição parecida com sua representação.

Os alunos se envolveram bastante com as atividades sobre perspectiva, tanto as realizadas no ambiente presencial como as do ambiente virtual. Alguns alunos apresentaram dificuldade para desenhar utilizando malha isométrica, e principalmente para fazer as representações dos sólidos no plano. Foi uma pequena parte que apresentou esse tipo de dificuldade. De acordo com Gutiérrez (1998), essas dificuldades ocorrem por envolver dois passos: interpretação da figura plana para convertê-la num objeto tridimensional e a interpretação desse objeto para convertê-lo no objeto geométrico de estudo. Acreditamos que, mesmo os alunos estando no ensino médio, essa dificuldade se apresenta devido a não realização deste tipo de ação em outros momentos da vida escolar, o que contribuiu para 
uma construção de imagens visuais ainda muito iniciais.

Enquanto essas atividades eram realizadas em sala de aula, no ambiente virtual os alunos resolviam problemas envolvendo visualização, que remetiam às atividades ora desenvolvidas, pois envolviam blocos construídos com cubinhos. Essas tarefas eram publicadas no ambiente virtual Facebook por meio de um arquivo em Word. Foram três problemas: A peça que falta, Cubos pintados, $O$ quebra-cabeça da letra $H$, retirados do livro Visualizando figuras espaciais, sob a coordenação de Segadas (2008), do projeto Fundão.

As discussões dos problemas citados, ocorridas por escrito no ambiente virtual, foram atividades ricas em interações e, consequentemente, em dados para análise. Na sequência, discutimos um desses problemas, a partir das interlocuções propiciadas pelos alunos.

\section{CURTINDO E COMENTANDO AS INTERAÇÕES DOS ALUNOS}

A análise das interações foi feita segundo as propriedades das interlocuções, apresentadas por Bairral e Powell (2013). A partir dessas interlocuções, identificamos habilidades do pensamento geométrico e, quando possível, estabelecemos uma conexão com a análise feita a partir dos questionários aplicados ${ }^{1}$.

A atividade selecionada é o problema "Cubos Pintados", representada pela figura 2.

1 Ao final da atividade prática desenvolvida na pesquisa de mestrado (ROVETTA, 2015), foi aplicado um questionário sobre o conteúdo estudo (sólidos geométricos), cujas questões foram utilizadas para análise, juntamente com as interações dos alunos em sala de aula e em rede social. 
Figura 2. Problema "Cubos Pintados".

\section{CUBOS PINTADOS}

Dezesseis cubos de $1 \mathrm{~cm}$ de lado săo colocados juntos, formando o paralelepípedo representado abaixo,

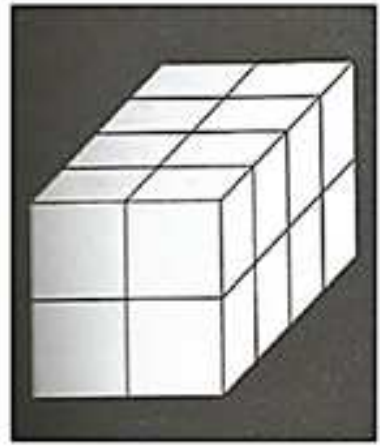

A superfície do mesmo foi pintada de verde e, em seguida, os cubos foram separados, Qual o número de cubos com exatamente duas faces verdes?

Fonte: Segadas (2008, p. 35).

$\mathrm{Na}$ resolução da atividade os alunos interagiram utilizando diferentes propriedades da interlocução. Nas interlocuções do quadro 1 observamos que os alunos limitaram-se a manifestar uma informação, sem explorá-la ou mesmo levantar outras discussões, portanto são interlocuções do tipo informativas.

Quadro 3. Interlocuções informativas.

\begin{tabular}{|c|c|}
\hline Alunos & Mensagens \\
\hline Jonas ${ }^{2} 8$ cubos ficarão com as duas faces pintadas de verde!! \\
\hline Wesley são 8 cubos com 2 faces pintadas \\
Maria 8 cubos com 2 faces \\
\hline
\end{tabular}

Fonte: dados da pesquisa.

Além da propriedade informativa, percebemos, no quadro 2, interlocuções interpretativas.

2 Pare preservar a identidade dos alunos, os nomes apresentados são fictícios. 
Quadro 3. Interlocuções interpretativas.

\begin{tabular}{|ll|}
\hline Alunos & \multicolumn{1}{c|}{ Mensagens } \\
\hline Fernanda & $\begin{array}{l}\text { Depende muito da interpretação! RS Então, acho que estou confusa para responder kk mas } \\
\text { analisando os comentários aqui deixados, de certo modo concordo que sejam } 8 \text { cubos, } 2 \text { faces } \\
\text { pintadas. }\end{array}$ \\
\hline $\begin{array}{l}\text { Isabel } \\
\text { Concordo com a Fernanda, fiquei meio confusa, mas depois de analisar os comentários, vi que } \\
\text { realmente teriam } 8 \text { cubinhos com } 2 \text { faces pintadas }\end{array}$ \\
\hline
\end{tabular}

Fonte: dados da pesquisa.

Já na interação apresentada no quadro 3, os alunos Euller, Janaína e Jair interagem mutuamente buscando a solução de um problema: a possibilidade de haver cubinhos com uma única face pintada de verde. Esses alunos estão abordando e desenvolvendo a interpretação figurativa que, de acordo com Gutiérrez (1996), identifica propriedades a partir da visualização do objeto. Essa interpretação e suas discussões são apresentadas nas interações que são bem expressivas e, nas quais, percebemos a predominância da interlocução negociativa, pois os interlocutores buscam coletivamente uma solução.

Quadro 3. Interações entre os alunos.

\begin{tabular}{|c|c|}
\hline Alunos & Mensagens \\
\hline Déby & $\begin{array}{l}\text { Eu acho que são } 8 \text { cubinhos que irão ficar com somente } 2 \text { faces pintadas de verde, pois só as } \\
\text { duas carreiras é que ficarão com duas de suas faces verdes }\end{array}$ \\
\hline Valci & concordo com a Deby pois os cantos tem 3 faces que foram pintadas!!! \\
\hline Janaína & $\begin{array}{l}\text { concordo com a Deby, serão } 8 \text { cubinhos com duas faces verdes, pois os outros serão de uma } \\
\text { ou três faces pintadas. }\end{array}$ \\
\hline Euller & $\begin{array}{l}\text { exato, os quadrados pintados que estão no meio do paralelepípedo são os que terão } 2 \text { faces } \\
\text { pintadas, ou seja, oito, porem só para fazer uma correção Janaina acredito que não haverá } \\
\text { quadrados com apenas uma face pintada. }\end{array}$ \\
\hline Janaína & $\begin{array}{l}\text { Depende, se a parte de baixo também for pintada, realmente não terão quadrados com } \\
\text { apenas uma face pintada. }\end{array}$ \\
\hline Jair & $\begin{array}{l}\text { Também concordo que seja oito quadrados assim como os outros, pois ao pintarmos a } \\
\text { superfície só os cubos do meio é que ficarão com duas de suas faces pintadas de verde. Os do } \\
\text { canto terão três faces pintadas de verde. Portanto nenhum dos cubos terão apenas uma face } \\
\text { pintada viu Janaína. }\end{array}$ \\
\hline Janaína & Tá ok. Apenas interpretei de outro jeito! Kkkk \\
\hline Euller & $\begin{array}{l}\text { se a parte de baixo não for pintada o resultado vai ser } 8 \text {, porem vai haver quadrados com só } \\
\text { um lado pintado }\end{array}$ \\
\hline Janaína & Disso que eu tava falando kkk \\
\hline
\end{tabular}


Euller porem Jair depende da interpretação, pois se consideramos a superfície como todos os lados que se pode ver em qualquer situação seu pensamento esta correto, porem se imaginarmos q superfície representa os lados visíveis no momento então o "Teorema da Janaina" está correto.

Fonte: dados da pesquisa.

No início da interação, os alunos Valci e Janaína, manifestam-se mostrando concordância com a resposta dada pela Déby. Porém, ambos não se limitam a concordar, mas também emitem um julgamento afim de aprofundar o raciocínio. Da mesma forma Euller se manifesta pontuando o que considera certo e errado na resposta dada pela Janaína, porém essa interlocução não é avaliativa, pois ele também manifesta argumentos aprofundando o raciocínio ao explicar que "os quadrados pintados que estão no meio do paralelepípedo são os que terão 2 faces pintadas".

Ainda em sua primeira fala, Janaína diz que os demais cubinhos terão três ou uma face pintada de verde, gerando assim outra discussão com a observação feita por Euller: "Acredito que não haverá quadrados com apenas uma face pintada". Daí em diante ele, a Janaína e o Jair, iniciam uma discussão a respeito da possibilidade de haver cubinhos com uma única face pintada, predominando assim a interlocução negociativa. Apesar de predominar o processo negociativo, outras interlocuções também são observadas. Por exemplo, quando Janaína responde "ta ok. Apenas interpretei de outro jeito! Kkk" ela apenas emite uma informação, caracterizando uma interlocução informativa.

Constatamos assim que interlocuções do tipo negociativa nos permitem analisar de forma mais ampla os seus interlocutores, indo além daquilo que eles escreveram. Isso vem ao encontro do que Bairral e Powell $(2013$, p.66) explicam sobre esse tipo de interlocução: "participantes engajados em interlocuções negociativas têm o potencial de desenvolver conjuntamente ideias matemáticas e formas de raciocínio que emergem nos discurso dos interlocutores $[\ldots] "$.

Isso se aplica na interlocução negociativa apresentada pelos alunos Euller, Jair e Janaina, de 
onde emergem considerações importantes sobre o pensamento geométrico como, por exemplo, habilidades que ainda estão em processo de construção. Apesar de fazer análises pertinentes ao contexto da discussão, Euller comete um erro ao chamar os cubos de quadrados. Com isso, percebemos que ainda há uma confusão na maneira de falar sobre formas plana e espacial. Essa confusão também está presente na escrita da aluna Janaína que na primeira vez utilizou o termo cubinhos e na segunda, após ler o argumento do Euller, ela também utiliza quadrados. Jair comete o mesmo erro. Como foi uma interlocução escrita não podemos afirmar que eles erram por não saber. Pensamos que eles sabem diferenciar quadrado de cubo, porém, notamos que no processo da linguagem esses termos ainda são trocados. Se fosse uma atividade presencial poderíamos questionar essa fala dos alunos.

Esse tipo de erro foi recorrente por mais alunos em outras atividades. Observamos isso ao analisar um dos questionários que foi aplicado durante o processo de investigação. A figura 3 mostra uma dessas questões.

Figura 4. Questão do questionário.

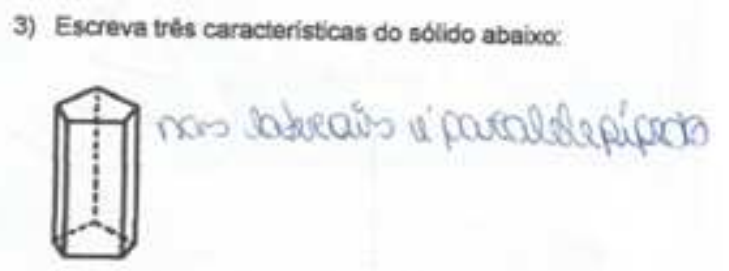

Fonte: acervo das autoras.

O aluno diz que as laterais do prisma são paralelepípedo ao invés de retângulos ou mesmo paralelogramos. Além do erro mostrado na figura 3, também encontramos outros erros relacionados à nomenclatura: é um hexaedro, apresenta 5 faces triangulares e 2 bases hexagonais; é um tetraedro; apresenta base retangular. Notamos que o desenvolvimento da linguagem matemática também é um processo a ser construído junto com o pensamento geométrico. Essa construção requer tempo e precisa ser trabalhada em diferentes ações pelo professor. Os exemplos citados representam formas escritas com trocas de nomenclatura, isso também foi percebido em sala de aula, mas a professora interferia questionando a 
linguagem, o que é uma limitação no momento da escrita. Verificamos, portanto, que se faz necessário um trabalho em longo prazo com diferentes atividades que ajudem a desenvolver a visualização e a compreensão da linguagem matemática própria, contribuindo assim para a construção do pensamento geométrico.

Ao escolher um modelo físico e gráfico para representar determinado conceito matemático, Gutiérrez (1998) destaca que é importante levar em consideração conhecimentos prévios dos alunos para ter o cuidado de selecionar um modelo adequado. Desse modo, as representações que são complexas para os alunos, que só transmitem conceitos de forma parcial, devem ser evitadas. Esse problema está presente quando temos que representar objetos geométricos tridimensionais por figuras planas. Portanto, "[...] a escolha de um bom modelo de representação plana de um sólido é essencial para o ensino e aprendizagem de geometria espacial” (GUTIÉRREZ, 1998, p. 194, tradução nossa).

Dessa forma, entendemos que desenhar e compreender as representações planas dos objetos tridimensionais facilita a aprendizagem da geometria espacial e melhora a sua capacidade de compreensão. Para isso, é importante que o professor acompanhe a evolução das habilidades de utilização de representações técnicas, visando evitar acúmulo de dificuldades e um possível bloqueio com a geometria.

Cabe ressaltar que essas atividades foram discutidas em aulas de matemática, presenciais e virtuais. Os dois ambientes tiveram a mesma relevância para o processo de ensino e aprendizagem. A relação entre os ambientes foi estabelecida de acordo com as interações que surgiram. Em determinados momentos, por exemplo, foi necessário levar certas discussões do ambiente virtual para sala de aula, a fim de explorá-las com maior profundidade. Já em outros momentos, discussões que surgiram em sala de aula foram ampliadas no ambiente virtual, a fim de oportunizar mais um momento de reflexão para o aluno. 


\section{COMPARTILHANDO CONCLUSÕES}

A aprendizagem matemática propiciada com a utilização de recursos tecnológicos variados, agregados aos espaços virtuais de aprendizagem, pode contribuir para um processo autônomo, construtivo e reflexivo de ensino e aprendizagem. As informações que num primeiro momento são recebidas por meio dos ambientes virtuais podem ser enriquecidas pelas informações que são compartilhadas com os demais, é por meio dessas interações que o conhecimento é construído e se compreende a importância do diálogo. Obviamente, não são todos os tipos de ambientes virtuais que possibilitam a aprendizagem por meio dessa interação dialógica. Há espaços como chats e fóruns, por exemplo, onde é possível desenvolver discussões sobre conceitos matemáticos de forma coletiva, criando assim uma comunidade de aprendizagem. É certo que cada um fará um tipo de construção do pensamento geométrico de acordo com sua interação com o grupo e processo da própria aprendizagem. Assim, notamos que esses espaços são propícios para este tipo de interação e que os diálogos podem contribuir para esse processo de aprendizagem.

Práticas como a apresentada mostram que é possível estabelecer uma conexão entre sala de aula e rede social interferindo positivamente na aprendizagem dos alunos. Além do mais, as interações desenvolvidas no ambiente virtual permitem ao professor analisar o desenvolvimento e aprofundamento do raciocínio matemático dos alunos. Destacamos que alguns alunos se sentiram mais à vontade para interagir no ambiente virtual, seja por timidez ou por outro motivo. Portanto, proporcionar diferentes espaços de interação nos ajuda a ampliar possibilidade para os diferentes alunos.

Constatamos a importância dos aspectos visuais no ensino da geometria, pois mesmo na fase final do ensino médio, grande parte do público pesquisado ainda não tinha habilidades básicas de visualização desenvolvidas. Percebemos também a importância do discurso e da interação para o aprendizado matemático, reforçando assim o que indica Bairral (2007). Além do mais, foi possível concluir que no processo inicial de construção do pensamento 
geométrico, os alunos classificam um sólido por sua aparência global e que, aos poucos começam a recorrer às características de um sólido a fim de identificá-lo.

Reconhecemos que, com esta pesquisa, não podemos generalizar a questão da utilização das redes sociais na educação, contudo ela traz indicativos de que, quando estabelecida uma conexão com as atividades desenvolvidas em sala de aula, a interação, a aprendizagem e o envolvimento dos alunos são satisfatórios.

\section{REFERÊNCIAS}

BAIRRAL, M. A. POWELL, A.B. Interlocuções e saberes docentes em interações on-line: um estudo de caso com professores de Matemática. Pro-posições, v. 24, n.1 (70), p.61-77. JAN/ABR 2013.

BAIRRAL, M.A. Discurso, interação e aprendizagem matemática em ambientes virtuais a distância. Rio de Janeiro: Edur, 2007.

BORBA, M.C.; SILVA, R.S.R.; GADANIDIS, G. Fases das tecnologias digitais em Educacação Matemática: sala de aula e internet em movimento. 1.ed. Belo Horizonte: Antência, 2014.

CARVALHO,M.L.O. Representações planas de corpos geométricos tridimensionais: uma proposta de ensino voltada para a codificação e decodificação de desenhos. 2010, $244 f$. Dissertação (Mestrado Profissional em Educação matemática). Universidade Federal de Ouro Preto. Ouro Preto, 2010.

GUTIERREZ, A. Lasrepresentaciones planas de cuerpos 3-dimensionales em La ensenanza de La geometria espacial. Revista EMA3.3, p. 193-220. Colômbia, 1998. Disponível em:<http://www.uv.es/angel.gutierrez/marcotex.html>. Acessoem: 20 mar. 2014.

GUTIERREZ, A. Procesos y habilidades em visualizacion espacial. Em: 3er Congresso Internacional sobre investigação em educação matemática, p. 44-59. Valência, 1991. Disponível em: <http://www.uv.es/angel.gutierrez/marcotex.html>. Acesso em: 13 mar. 2015.

GUTIERREZ, A. Visualization in 3-dimensional geometry: in search of a framework. Em: PUIG,L; GUTIÉRREZ, A. Proceedings of 20th PME Conference, v.3, p.19-26,Universidade de Valência, Spain, 1996. 
GUTIÉRREZ, A.; JAIME, A.; FORTUNY, J.M. An alternative paradigm to evaluate the acquisition of the Van HieleLevels. In: Journal for Research in Mathematics Education. v. 22, n. 3, p. 237251.1991.

KALEFF, A.M.M.R. Vendo e entendendo os poliedros. 2. ed. Rio de Janeiro, 2003.

MIGUEL, A.; FIORENTINI, D.; MIORIM, M. A. Álgebra ou Geometria: para onde pende o pêndulo? ProPosições. São Paulo, v.3, n.1 (7), p.39-54. Março, 1992.

NACARATO, A.M.; SANTOS, C.A. Aprendizagem em Geometria na educação básica: a fotografia e a escrita na sala de aula. 1.ed. Belo Horizonte: Autêntica, 2014.

NASSER, L.; TINOCO, L. (Coord). Curso Básico de Geometria: formação de conceitos geométricos. Rio de Janeiro: Ed. IM/UFRJ, 2011.

POWELL, A. B. Socially emergent cognition: particular outcome of student-to-student discursive interactions during mathematical problem solving. Horizontes, v.21, n.1, p.33-42. 2006.

ROVETTA, O.M. Interações em sala de aula e em redes sociais no estudo de sólidos geométricos no ensino médio. 2015, 166 f. Dissertação (Mestrado Profissional em Educação em Ciências e Matemática). Instituto Federal do Espírito Santo, Vitória, 2015.

SEGADAS, C. (Coord.). Visualizando Figuras Espaciais. Rio de Janeiro: IM/UFRJ, 2008. 